\title{
Aspectos \\ epidemiológicos \\ de \\ casos \\ de loxoscelismo registrados en Direcciones de Salud de Lima y Direcciones Regionales de Salud de Perú. Periodo 2009-2018
}

Epidemiological aspects of loxoscelism cases reported in the Control and Prevention Disease National Center of Peru. 2009 to 2018 period

\author{
Alejandra Vega ${ }^{1}$, Daphne León ${ }^{2}$, Oswaldo Cabanillas ${ }^{3}$, Néstor Falcón ${ }^{2}$
}

\section{RESUMEN}

El objetivo del estudio fue describir el comportamiento espacial y temporal de los casos de loxoscelismo registrados en la sala virtual de situación de salud del Centro Nacional de Epidemiología, Prevención y Control de Enfermedades (CDC-Perú) en el periodo 2009 - 2018. Se recolectó información correspondiente a las variables grupo etario, sexo, año y región geográfica en el que se reportaron las mordeduras. Con la información recopilada se creó una base de datos en Microsoft Excel la que fue resumida mediante estadística descriptiva (frecuencias absolutas y relativas). Se observó una tendencia creciente en el reporte de accidentes loxoscélicos, alcanzando el máximo número en el año 2016. El grupo etario más afectado fue el de 30 a 59 años (4 108 casos) seguido del de 18 a 29 años (2 014 casos). En general, las mujeres fueron las más afectadas (5 265 casos), pero en la población infantil, los casos se reportaron con mayor frecuencia en los varones. Lima fue la región con mayor número de reportes (2 958 casos), no obstante, la región con mayor tasa de presentación fue Arequipa con 150,9 casos por cada 100000 habitantes. El estudio mostró que en regiones y temporadas de mayor temperatura los casos de loxoscelismo aumentaban. Además, se observó que los accidentes loxoscélicos pueden ocurrir a cualquier edad por diversos factores de exposición, por lo que sería importante implementar campañas de prevención orientadas a disminuir el riesgo de exposición en las diferentes etapas de la vida de las personas.

PALABRAS CLAVE: Loxoscelismo, arácnidos, epidemiología.

\section{SUMMARY}

The objective was to describe the spatio-temporal behavior of loxoscelism cases reported in the health situation virtual space from the "Centro Nacional de Epidemiología, Prevención y Control de Enfermedades" (CDC-Perú) since 2009 until 2018. We gathered information about the age group, sex, year and geographical region where the bite was reported. With the collected information the data base was created in Microsoft Excel and was summarized by using descriptive statistics (absolute and relative frequencies). The number of loxoscelism reported cases had a growing tendency and reached its peak in 2016. The most affected age group was the one from 30 to 59 years old (4 108 cases) followed by the one from 18 to 29 (2 014 cases). Women were the most affected sex from the whole population (5 265 cases) but among the infant population the male sex was the most affected. Lima was the region

\footnotetext{
Grupo de Salud Pública Veterinaria (SAPUVET-PERÚ). Lima, Perú.

2 Laboratorio de Epidemiología y Salud Pública en Veterinaria, Facultad de Medicina Veterinaria y Zootecnia, Universidad Peruana Cayetano Heredia. Lima, Perú.

3 Centro Nacional de Epidemiología, Prevención y Control de Enfermedades (CDC). Lima, Perú.
} 
with the highest number of cases reported (2 958 cases) however the region with the highest presentation rate was Arequipa with 150.9 cases per 100000 population. This study showed that in regions and seasons with higher temperatures the number of loxoscelism cases was higher. Also, loxoscelism could happen at any age because of different risk factors and that is why it would be important to create prevention campaigns that specify the risk factors at each life stage.

KEYWORDS: Loxoscelism, arachnids, epidemiology.

\section{INTRODUCCIÓN}

La mayoría de accidentes por animales ponzoñosos suelen ser causados por arácnidos (Segura, Hernández, Falcón, y Silva, 2013). Las arañas son artrópodos pertenecientes a la Clase Arachnida y la mayoría produce veneno para adormecer $\mathrm{y} / \mathrm{o}$ predigerir a sus presas, aunque no todas son perjudiciales para el ser humano (Instituto Nacional de Salud, 2004; Haas et al., 2012).

Las Organización Mundial de la Salud (OMS) considera de interés médico a aquellas pertenecientes a los géneros Loxosceles, Latrodectus, Phoneutria y Lycosa debido a la peligrosidad de su veneno y a los signos clínicos que producen en los seres humanos (Almada y Medrano, 2006), siendo la especie Loxosceles laeta la más común en Perú, Argentina, Chile y el sur de Brasil (Schenone, 1998; Cabrerizo et al., 2009). Según el Ministerio de Salud (MINSA), esta especie se encuentra ampliamente distribuida en zonas urbanas y urbano-marginales de la costa y sierra de Perú (Ministerio de Salud, 2004).

L. laeta no es agresiva y suele vivir en lugares oscuros y apartados (Málaque et al., 2002). Se puede encontrar en partes oscuras de la casa donde la limpieza es poco frecuente como rincones, guardarropas, hendiduras en paredes, etc., y en el exterior de las viviendas debajo de troncos, piedras, entre otros (Miranda, Velarde, Beltrán y Núñez, 2006). Es sedentaria y suele ser más activa durante la noche (Haas et al., 2012). Tejen telas algodonosas e irregulares en los lugares que habitan y se alimentan de moscas, polillas y otros artrópodos (Zavaleta, 1987; Miranda et al., 2006). Pueden sobrevivir meses sin agua o comida y resisten temperaturas de $8{ }^{\circ} \mathrm{C}$ a $43{ }^{\circ} \mathrm{C}$ (Appel, da Silveira, Gremski y Veiga, 2005).

El loxoscelismo produce impacto en la salud pública ocasionado por el alto índice de infestación intradomiciliaria que se presenta en ciudades costeras y debido a los cuadros clínicos que produce (Zavaleta,
1987). En este contexto, el estudio tuvo como objetivo describir el comportamiento epidemiológico, espacial y temporal, de casos de loxoscelismo registrados en la sala virtual de situación de salud del Centro Nacional de Epidemiología, Prevención y Control de Enfermedades (CDC-Perú) en el periodo 2009 - 2018.

\section{MATERIAL Y MÉTODOS}

El estudio se realizó en la Facultad de Medicina Veterinaria y Zootecnia de la Universidad Peruana Cayetano Heredia (FAVEZ-UPCH). El estudio correspondió a una investigación observacional, descriptiva y retrospectiva.

La población objetivo estuvo constituida por los casos notificados de loxoscelismo que fueron reportados por las Direcciones de Redes Integradas de Salud (DIRIS), las Direcciones Regionales de Salud (DIRESAS) y las Gerencias Regionales de Salud (GERESAS) de Perú durante el periodo 2009-2018. Esta información se recuperó de la Sala Virtual de Situación de Salud del Centro Nacional de Epidemiología, Prevención y Control de Enfermedades (CDC-Perú), la misma que es de acceso libre y se encuentran a disposición del público en general a partir de la página web del CDC-Perú en el siguiente link: https://www.dge.gob.pe/salasituacional/

Se recolectó la información del grupo etario, sexo, año, estación (considera una distribución arbitraria al dividir el año en cuartiles) y región de reporte. La información recopilada se registró en una hoja de cálculo en el programa Microsoft Excel 2016. Los resultados se resumieron mediante estadística descriptiva (frecuencias absolutas y relativas) y se presentan en cuadros y gráficos. La tasa de presentación de casos para el periodo de estudio se calculó utilizando como población humana de referencia la del año 2015 (Instituto Nacional de Estadística e Informática (INEI), 2015). La diferencia entre la presentación de casos según sexo se evaluó mediante la prueba de Ji Cuadrado. 
El estudio tuvo el aval del Comité Institucional de Ética de la Universidad Peruana Cayetano Heredia mediante la Constancia 266-12-18.

\section{RESULTADOS}

El estudio recolectó información de $10 \quad 131$ accidentes loxoscélicos registrados en las DIRIS, DIRESAS y GERESAS, durante el periodo 20092018; en la tabla 1 se muestra la distribución de los casos según año y grupo etario afectado, mientras que en la tabla 2 se presentan los casos de loxoscelismo distribuidos según año de registro y sexo del afectado. La distribución de los casos de loxoscelismo según estación y año de registro se presenta en el gráfico 1.

Se observa que la región donde se registró el mayor número de accidentes por loxoscelismo fue Lima con un total de 2958 casos $(29,2 \%)$, seguido por Arequipa con 1943 casos (19,18\%) y La Libertad con 1075 casos (10,61\%). También se observó que Arequipa es la región con mayor tasa de presentación de casos de loxoscelismo en el período 2009-2018 con 150,9 casos por cada 100000 habitantes, seguido por

Tabla 1. Casos de loxoscelismo distribuidos según año de registro y grupo etario del afectado en Perú durante el periodo 2009-2018

\begin{tabular}{ccccccccc}
\hline Año & Menos de 1 & $\mathbf{1 ~ a ~ 4 ~}$ & $\mathbf{5}$ a 11 & $\mathbf{1 2}$ a 17 & $\mathbf{1 8}$ a 29 & $\mathbf{3 0}$ a 59 & $\mathbf{6 0}$ a más & TOTAL \\
\hline 2009 & 10 & 39 & 44 & 55 & 104 & 206 & 46 & 504 \\
2010 & 3 & 61 & 52 & 70 & 151 & 256 & 60 & 653 \\
2011 & 3 & 50 & 59 & 59 & 130 & 253 & 74 & 628 \\
2012 & 3 & 56 & 58 & 79 & 152 & 281 & 86 & 715 \\
2013 & 7 & 53 & 71 & 51 & 159 & 298 & 79 & 718 \\
2014 & 5 & 94 & 94 & 96 & 189 & 351 & 129 & 958 \\
2015 & 10 & 59 & 72 & 62 & 150 & 278 & 87 & 718 \\
2016 & 11 & 198 & 224 & 163 & 360 & 803 & 236 & 1995 \\
2017 & 11 & 156 & 167 & 135 & 337 & 721 & 224 & 1751 \\
2018 & 15 & 104 & 125 & 112 & 282 & 661 & 192 & 1491 \\
Total & 78 & 870 & 966 & 882 & 2014 & 4108 & 1213 & 10131 \\
\hline
\end{tabular}

Fuente: Centro Nacional de Epidemiología, Prevención y Control de Enfermedades (CDC-Perú), 2019.

Tabla 2. Casos de loxoscelismo distribuidos según año de registro y sexo del afectado en Perú durante el periodo 2009-2018.

\begin{tabular}{ccccccc}
\hline \multirow{2}{*}{ Añ } & \multicolumn{2}{c}{ Femenino } & & \multicolumn{2}{c}{ Masculino } \\
\cline { 2 - 3 } \cline { 5 - 6 } & $\mathbf{n}$ & $\mathbf{\%}$ & & $\mathbf{n}$ & $\mathbf{\%}$ \\
\hline 2009 & 254 & 4,8 & & 250 & 5,1 \\
2010 & 325 & 6,2 & & 328 & 6,7 \\
2011 & 308 & 5,8 & & 320 & 6,6 \\
2012 & 352 & 6,7 & & 363 & 7,5 \\
2013 & 364 & 6,9 & & 354 & 7,3 \\
2014 & 477 & 9,1 & & 481 & 9,9 \\
2015 & 397 & 7,5 & & 321 & 6,6 \\
2016 & 1092 & 20,7 & & 903 & 18,6 \\
2017 & 905 & 17,2 & & 846 & 17,4 \\
2018 & 791 & 15 & & 700 & 14,4 \\
Total & 5265 & 100 & & 4866 & 100 \\
\hline
\end{tabular}

Fuente: Centro Nacional de Epidemiología, Prevención y Control de Enfermedades (CDC-Perú), 2019 


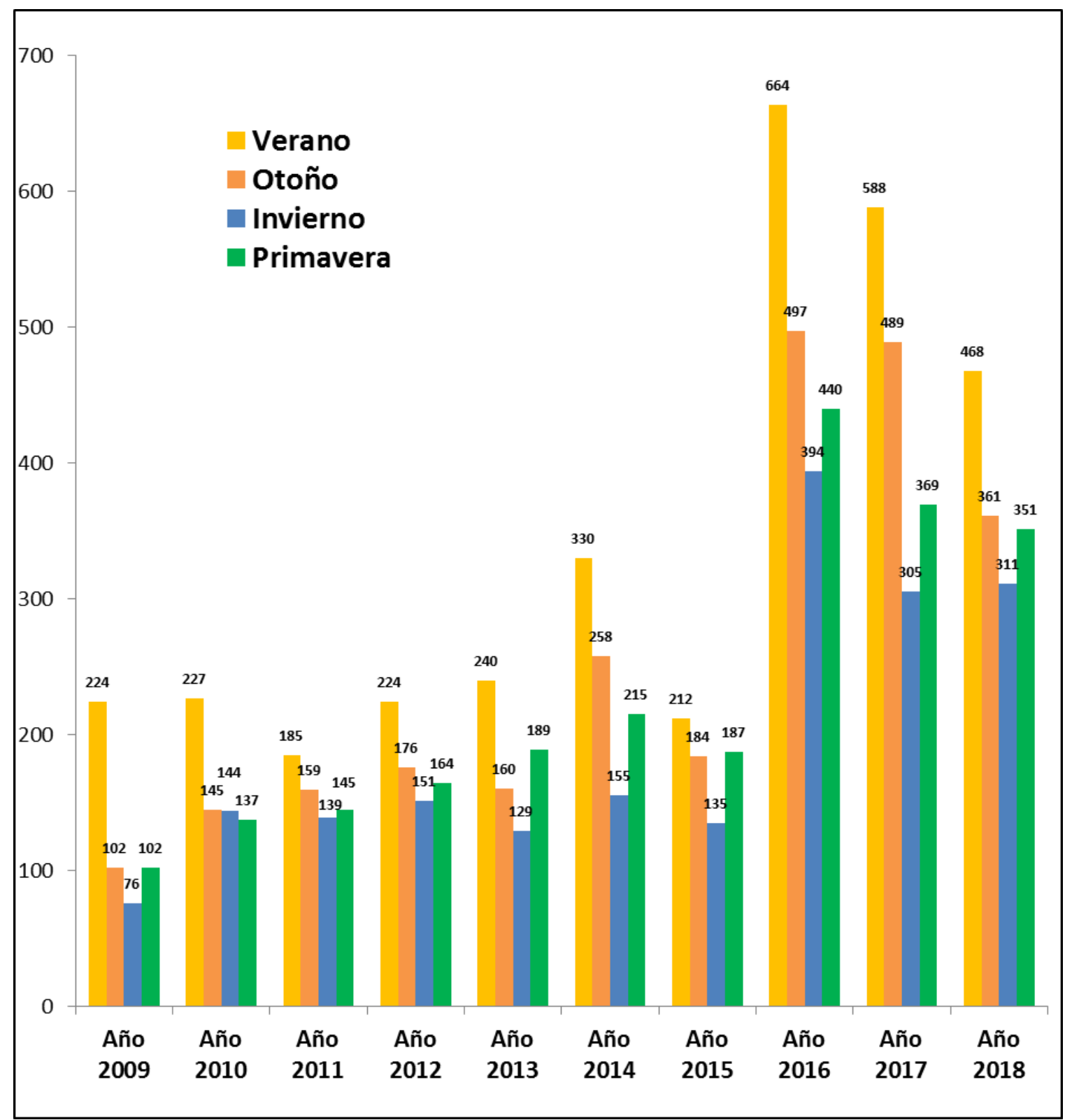

Gráfico 1. Casos de loxoscelismo distribuidos según año y estación, registrados en Perú durante el periodo 2009-2018 (Fuente: Centro Nacional de Epidemiología, Prevención y Control de Enfermedades (CDC-Perú), 2019)

Amazonas con 93,5 y Ayacucho con 73,6. El detalle de la distribución de los casos expresados en valores absolutos y relativos se presenta en la tabla 3.

\section{DISCUSIÓN}

La notificación de casos de loxoscelismo ha seguido una tendencia creciente desde el año 2009, alcanzando el mayor número de reportes el año 2016. Esto se podría deber a que en ese año y en el periodo de junio a diciembre hubo un calentamiento anómalo en la costa de Perú que antecedió al fenómeno del Niño Costero 2017 (Takashi, 2017). Durante las semanas de invierno del 2016 el número de casos se mantuvo elevado en comparación a lo observado en otros años. Schenone, Saavedra, Rojas y Villarroel (1989) mencionan que existe una relación directamente proporcional entre la temperatura ambiental y la incidencia de loxoscelismo debido al hallazgo de más casos en estaciones más calurosas.

Las estaciones del año en que se presentaron más casos de loxoscelismo fueron verano y otoño. Este resultado difiere de los hallazgos de estudios realizados en el extranjero como el de Schenone et al., (1989) realizado en Chile y el de Málaque et al., (2002) 
Tabla 3. Distribución proporcional y tasa de presentación de los casos de loxoscelismo según región de registro. Periodo 2009-2018.

\begin{tabular}{|c|c|c|c|c|}
\hline \multirow[t]{2}{*}{ Región } & \multicolumn{2}{|c|}{$\begin{array}{c}\text { Distribución Proporcional de } \\
\text { Casos }\end{array}$} & \multicolumn{2}{|c|}{$\begin{array}{c}\text { Tasas de Presentación (Casos/100 } 000 \\
\text { habitantes) }\end{array}$} \\
\hline & $\mathrm{n}^{(1)}$ & $\%$ & Población $2015^{(2)}$ & Tasa \\
\hline Lima & 2958 & 29,20 & 9838251 & 30,1 \\
\hline Arequipa & 1943 & 19,18 & 1287205 & 150,9 \\
\hline La Libertad & 1075 & 10,61 & 1859640 & 57,8 \\
\hline Ayacucho & 507 & 5,00 & 688657 & 73,6 \\
\hline Junín & 423 & 4,18 & 1350783 & 31,3 \\
\hline San Martin & 397 & 3,92 & 840790 & 47,2 \\
\hline Amazonas & 395 & 3,9 & 422629 & 93,5 \\
\hline Piura & 363 & 3,58 & 1844129 & 19,7 \\
\hline Huánuco & 349 & 3,44 & 860537 & 40,6 \\
\hline Callao & 335 & 3,31 & 1010315 & 33,2 \\
\hline Cusco & 329 & 3,25 & 1316729 & 25,0 \\
\hline Huancavelica & 191 & 1,89 & 494963 & 38,6 \\
\hline Ancash & 189 & 1,87 & 1148634 & 16,5 \\
\hline Tacna & 186 & 1,84 & 341838 & 54,4 \\
\hline Cajamarca & 176 & 1,74 & 1529755 & 11,5 \\
\hline Loreto & 79 & 0,78 & 1039372 & 7,6 \\
\hline Puno & 53 & 0,52 & 1415608 & 3,7 \\
\hline Lambayeque & 49 & 0,48 & 1260650 & 3,9 \\
\hline Ica & 43 & 0,42 & 787170 & 5,5 \\
\hline Apurímac & 32 & 0,32 & 458830 & 7,0 \\
\hline Pasco & 25 & 0,25 & 304158 & 8,2 \\
\hline Tumbes & 17 & 0,17 & 237685 & 7,2 \\
\hline Ucayali & 15 & 0,15 & 495522 & 3,0 \\
\hline Madre de Dios & 2 & 0,02 & 137316 & 1,5 \\
\hline Total & 10131 & 100,0 & 30971166 & 32,7 \\
\hline
\end{tabular}

Fuente: ${ }^{1 .}$ Centro Nacional de Epidemiología, Prevención y Control de Enfermedades (CDC-Perú), 2019; ${ }^{2}$. Instituto

Nacional de Estadística e Informática (INEI), 2015.

realizado en Brasil, en los que observaron que el mayor número de casos se presentó en verano y primavera. Sin embargo, la presencia de temperatura elevada ha de ser más importante que la estación per se por lo que el aumento de casos se debe a que la temperatura en la costa peruana es más elevada durante los meses de otoño que durante primavera. Mientras que en otoño la temperatura puede alcanzar $27^{\circ} \mathrm{C}$, en primavera la temperatura alcanza un máximo de $24^{\circ} \mathrm{C}$ (Climate Data Organization, 2019a).

Las regiones donde se registraron más casos de loxoscelismo fueron Lima, Arequipa y La Libertad. Lima es la región que tuvo mayor número de reportes, aunque si se considera la tasa de presentación de estos accidentes, su ubicación dentro de las zonas más afectadas disminuye. La presencia de una mayor notificación podría estar relacionado a los siguientes factores: a). el número de casos podría estar relacionado a que es la región más poblada en el Perú y por ello tal circunstancia ofrece más espacios donde las arañas pueden habitar (INEI, 2018), b). la presencia de un rango de temperatura favorable para la L. laeta a lo largo año, por lo que se puede mantener activa durante todo este tiempo (Climate Data Organization, 2019b), c). la notificación también se vería aumentada debido a que Lima cuenta con más Direcciones de Salud que cualquier otra región y d). 
por la accesibilidad para la notificación y atención médica la que sería más fácil debido al desarrollo vial (Centro Nacional de Epidemiología, Prevención y Control de Enfermedades (CDC-Perú), 2019).

En comparación a estudios reportados en años anteriores, se observa que La Libertad desplaza de los primeros lugares de notificación de accidentes loxoscélicos al Cusco (INS, 2004). El hecho de que La Libertad ahora sea la tercera región más afectada por casos de loxoscelismo puede deberse al aumento poblacional que ha tenido en los últimos años, convirtiéndose en la tercera región más poblada del Perú según el último censo del Instituto Nacional de Estadística e Informática (INEI, 2018). L. laeta es una especie que habita en el interior de las viviendas, por lo que el aumento poblacional le ofrecería mayor oportunidad de hábitats $\mathrm{y}$ mayor posibilidad de contacto araña-humano.

En contraste con el número de notificaciones de accidentes por loxoscelismo, Arequipa, Amazonas y Ayacucho se encuentran entre las regiones con mayor tasa de presentación de casos en el periodo de estudio. Según el estudio epidemiológico hecho por Miranda et al., (2006), Arequipa, Caravelí y Castilla son las provincias de Arequipa con mayor número de casos reportados de loxoscelismo. Estas tres provincias cuentan con zonas urbanas y urbano-marginales, además de un clima que se encuentra dentro del rango de temperatura ideal para la L. laeta durante todo el año (Climate Data Organization, 2019a).

Madre de Dios es la región con menor tasa de presentación de loxoscelismo. Esto se debería a que esta región alcanza temperaturas de $36^{\circ} \mathrm{C}$ lo cual está en el límite superior del rango de temperatura ideal para L. laeta (Santos, 2017), a lo que se sumaría la presencia en la selva de arañas Scytodes globula, las cuales son depredadores naturales de la L. laeta. Según Alfaro, Veloso, Torres-Contreras, Solis y Canals (2013) ambas especies optan por microhabitats similares y prefieren las temperaturas bajas del amanecer por lo que habría una gran probabilidad de que coincidan y exista predación lo cual se vería reflejado en la epidemiología del loxoscelismo.

Es importante presentar la información tanto en número de casos como en tasa de presentación. El primero ayuda a tomar medidas de tipo logístico como calcular la cantidad de suero antiloxoscélico que se necesita abastecer para cubrir la demanda. El segundo permite visualizar el impacto que tiene en la población desde un punto de vista epidemiológico. Ambas mediciones son importantes y han de permitir el desarrollo de actividades que eviten el impacto negativo del loxoscelismo en la salud de las personas.

El sexo femenino fue el más afectado. Este hallazgo se repite en diversos estudios retrospectivos que han revisado historias clínicas de casos de loxoscelismo en hospitales como el de Núñez-Moscoso y ChacónArévalo (2013) o el de Rodríguez (2013). Sin embargo, Moranchel-García et al., (2017) consideran que el sexo no es un factor de riesgo para el loxoscelismo ya que esta especie es de amplia distribución y ambos géneros están igualmente expuestos. Además, las actividades que realizaban los afectados al momento del accidente según muestran los estudios son realizadas por ambos sexos como el vestirse, dormir y trabajar (Webb, Maguiña y González, 2010). Sin embargo, algunos factores culturales podrían contribuir a que el sexo femenino sea el más afectado. Por ejemplo, en algunos contextos sociales la limpieza es una actividad realizada generalmente por la mujer (pareja, personal de servicio, etc.), lo que puede representar un factor de exposición al no protegerse durante la limpieza de lugares donde habita L. laeta.

En los grupos etarios de 1 a 4,5 a 11 y 12 a 17 años se observa un aparente mayor número de pacientes masculinos que femeninos. Al respecto, Segura et al., (2013) observaron más casos en niños que en niñas pacientes pediátricos del Instituto Nacional de Salud del Niño y mencionó que el sexo podría ser un factor importante en los casos de aracneismo. Dicho estudio encontró que algunas historias clínicas reportaban que el niño había estado jugando con el arácnido antes del accidente. Además, considera que la edad es un factor de exposición debido a que los niños de menor edad tienden a jugar en posibles hábitats de arácnidos y no tienen conocimiento de las consecuencias que podría traer jugar con una araña.

El grupo etario más afectado fue el de 30 a 59 años seguido del de 18 a 29 años. Rodríguez (2013) encontró resultados comparables al revisar historias clínicas de pacientes atendidos en el Hospital Regional Docente de Trujillo que fueron dados de alta desde el 2008 hasta el 2012 y observó que el grupo más afectado fue el de 31 a 40 años seguido del grupo de 10 a 20 años.

Los niños y niñas menores de 1 año fueron los menos afectados por el loxoscelismo. Este grupo tendría acceso limitado a lugares donde habitan arañas como rincones, roperos, etc. Sin embargo, se exponen 
al gatear, al dormir y al cambiarles la ropa sin antes sacudirla, por lo que la prevención depende de los padres.

Se observaron casos en niños menores de 1 año hasta en adultos mayores de 60 años. Esto indica que a lo largo de la vida habría diversos factores que exponen a las personas al loxoscelismo. Según Segura et al., (2013), durante la infancia podría ser el juego en lugares donde habita la araña, mientras que Schenone et al. (1989), mencionan que en la adultez podría ser el dormir, limpiar o cambiarse de ropa.

Debido a que el loxoscelismo podría ocurrir en cualquier etapa de la vida y en situaciones cotidianas, es importante que los planes de educación sanitaria y las campañas de prevención sean dirigidos a personas de todas las edades y que se enfoquen en prevenir situaciones de riesgo que se presentan en cada etapa de la vida. Las recomendaciones generales incluyen el mantener muebles y camas alejados de las paredes, evitar el uso de clavos o percheros para colgar ropa en la pared, cuidar el manejo de los juguetes de niños pequeños y sacudir la ropa antes de usarla y las sábanas antes de acostarse en cama. A ello se suma el evitar que los niños se escondan debajo de camas o muebles al jugar y que no toquen ni jueguen con las arañas que encuentren.

En Perú los dos principales géneros de arácnidos de importancia médica son Loxosceles y Latrodectus. El loxoscelismo causa necrosis dérmica e insuficiencia renal que puede ser letal. Segura et al., (2013) hallaron 2 casos letales en su estudio. Por el contrario, no se han registrado muertes por latrodectismo en Perú (Maguiña, Figueroa y Pulcha, 2017). Sin embargo, se debe de tener en cuenta que el veneno de la Latrodectus tiene una dosis letal $50 \mathrm{de} 20 \mu \mathrm{g} / \mathrm{kg}$ de peso mientras que el veneno de la loxosceles tiene $200 \mu \mathrm{g} / \mathrm{kg}$ de peso; es decir el veneno latrodéctico es 10 veces más letal que el loxoscélico (Moranchel-García et al., 2017). Por ello, la vigilancia y medidas preventivas deben ser enfocadas a ambas especies.

El loxoscelismo puede generar un impacto sobre la economía de las familias debido a que el grupo más afectado estuvo constituido por la población económicamente activa, dado que el afectado tendría que gastar dinero en medicamentos y perdería días de trabajo si es internado. Núñez-Moscoso y ChacónArévalo (2013) hicieron una revisión de casos de loxoscelismo en el período 2008-2012 en el Hospital Edmundo Escomel EsSalud de Arequipa, encontrando que el tiempo promedio de estancia hospitalaria fue de 3 días con un rango de 1 a 6 días. Por otro lado, Mendoza y Cabezas (2006) mencionan que en algunos casos se han de requerir escarectomía o reparación de úlceras por lo que los pacientes han de requerir un determinado periodo de recuperación pos cirugía lo que implica perdidas de días de trabajo.

La información recopilada en el estudio describe el comportamiento epidemiológico del loxoscelismo en Perú. Se espera que estos resultados sirvan como base para desarrollar o reforzar planes de educación sanitaria y campañas de prevención dirigidos específicamente a los grupos de personas más afectados y que se enfoquen en prevenir situaciones de riesgo que se presenta en cada etapa de la vida de las personas.

\section{CONCLUSIONES}

El estudio recolectó información de 10131 casos confirmados de loxoscelismo que fueron reportados a las DIRIS, DIRESAS y GERESAS del Perú durante el periodo 2009-2018 y llega a las siguientes conclusiones:

- La mayor cantidad de casos se presentó durante temporadas de mayor temperatura, siendo Arequipa la región con mayor tasa de presentación de casos de loxoscelismo para el periodo de estudio.

- Existe exposición al loxoscelismo a lo largo de diferentes etapas de la vida, ya que se registraron casos de niños menores de 1 año hasta adultos mayores de 60 años.

- El loxoscelismo tiene impacto en la economía nacional ya que afecta al grupo etario que conforma la fuerza de trabajo por lo que es necesario implementar planes de educación sanitaria y campañas de prevención que especifiquen los factores de riesgo a los que están expuestos cada grupo etario.

\section{Correspondencia:}

Néstor Falcón

Correo electrónico: nestor.falcon@upch.pe

\section{REFERENCIAS BIBLIOGRAFICAS}

1. Alfaro, C., Veloso, C., Torres-Contreras, H., Solis, R. \& Canals, M. (2013). Thermal niche overlap of the corner recluse spider Loxosceles laeta (Araneae; Sicariidae) and its possible predator, the spitting spider Scytodes globula (Scytodidae). Journal of Thermal Biology, 38(8), 502-507.

2. Almada, M. \& Medrano, C. (2006). Guía didáctica 
de arañas. Santa Fe: Museo provincial de Ciencias Naturales Florentino Ameghino.

3. Appel, M., da Silveira, R., Gremski, W. \& Veiga, S. (2005). Insights into brown spider and loxoscelism. Invertebrate Survival Journal, 2(2), 152-158.

4. Cabrerizo, S., Docampo, P., Cari, C., Ortiz, M., Díaz, M., de Roodt, A. \& Curci, O. (2009). Loxoscelismo: epidemiología y clínica de una patología endémica en el país. Archivos argentinos de pediatría, 107(2), 152-159.

5. Centro Nacional de Epidemiología, Prevención y Control de Enfermedades. (2019). Sala virtual de situación de salud. Lima: Centro Nacional de Epidemiología, Prevención y Control de Enfermedades. . Disponible en: https://www.dge. gob.pe/salasituacional/

6. Climate Data Organization. (2019). Clima: Arequipa. Oedheim: Climate Data Organization. Recuperado de: https://es.climate-data.org/ america-del-sur/peru/arequipa-1050/

7. Climate Data Organization. (2019). Clima: Lima. Oedheim: Climate Data Organization. Recuperado de: https://es.climate-data.org/america-del-sur/ peru/lima/lima-1014/

8. Haas, A., Orduna, T., Lloveras, S., de Roodt, A., Costa, V. \& García, S. (2012). Guía de prevención, diagnóstico, tratamiento y vigilancia epidemiológica de los envenenamientos por arañas. Buenos Aires: Ministerio de Salud, Programa Nacional de Prevención y Control de las Intoxicaciones.

9. Instituto Nacional de Estadística e Informática. (2015). Perú: Sintesis estadística 2015.Lima: Instituto Nacional de Estadística e Informática.

10. Instituto Nacional de Estadística e Informática. (2018). Perú: Perfil sociodemográfico. Informe Nacional. 13-22. Lima: Instituto Nacional de Estadística e Informática.

11. Instituto Nacional de Salud. (2004). Diagnóstico $y$ tratamiento de accidentes por animales ponzoñosos. Lima: Centro Nacional de Productos Biológicos.

12. Maguiña, C., Figueroa, V. \& Pulcha, R. (2017). Actualización sobre manejo de araneismo en Perú. Rev Méd Hered, 28(3), 200-207.

13. Málaque, C., Castro-Valencia, J., Cardoso, J., FranÇa, F., Barbaro, K. \& Fan, H. (2002). Clinical and epidemiological features of definitive and presumed loxoscelism in Sao Paulo, Brazil. Revista do Instituto de Medicina Tropical de São Paulo, 44(3), 139-143.

14. Mendoza, C. \& Cabezas, C. (2006). Loxoscelismo: evaluación clínica, tratamiento y prevención. Revista Peruana de Enfermedades Infecciosas y Tropicales, 5(1), 2-8.
15. Ministerio de Salud. (2004). Norma técnica sobre prevención y tratamiento de accidentes por animales ponzoñosos. Lima, Perú: Ministerio de Salud. $1-58 \mathrm{pp}$.

16. Miranda, P., Velarde, J., Beltrán, S. \& Núñez, R. (2006). Información a la población y medidas de prevención y atención de accidentes por mordedura de arácnidos. Arequipa: Ministerio de Salud, Región de Salud de Arequipa, Oficina de Epidemiología.

17. Moranchel-García, L., Pineda-Galindo, L., Casarrubias-Ramírez, M., Mendoza-Álvarez, S., Olvera-Acevedo, A., Alfaro-Mejía, J...BriceñoMoya, F. (2017). Evolución clínica de pacientes con loxoscelismo sistémico y dermonecrótico en un hospital de tercer nivel. Medicina interna de México, 33(1), 18-27.

18. Núñez-Moscoso, L. \& Chacón-Arévalo, S. (2013). Aspectos clínicos y epidemiológicos del loxoscelismo en un hospital de primer nivel. Revista de la Sociedad Peruana de Medicina Interna, 26(1), 14.

19. Rodríguez, P. (2013). Características clínicasepidemiológicas, complicaciones y tratamiento del Loxoscelismo, Hospital Regional Docente de Trujillo. (Tesis de pre grado). Universidad Nacional de Trujillo, Trujillo, Perú.

20. Santos, D. (2017) Caracterización hidrológica de la región Madre de Dios. Lima, Perú: Servicio Nacional de Meteorología e Hidrología del Perú.

22. Schenone, H. (1998). Loxoscelismo cutáneo de predominio edematoso. Boletín chileno de parasitología, 53: 78-83.

23. Schenone, H., Saavedra, T., Rojas, A. \& Villarroel, F. (1989). Loxoscelism in Chile: epidemiological, clinical and experimental studies. Revista do Instituto de Medicina Tropical de São Paulo, 31(6), 403-415.

24. Segura, M., Hernández, H., Falcón, N. \& Silva, W. (2013). Accidentes por animales ponzoñosos en pacientes internados en un hospital de niños en Lima, Perú. Estudio retrospectivo en el periodo 2000-2009. Salud y Tecnología Veterinaria, 1, 5259 .

25. Takashi, K. (2017). Fenómeno El Niño: "Global" vs "Costero". Instituto Geofísico del Perú: Generación de información y monitoreo del Fenómeno El Niño Boletín Técnico, 4(4).

26. Webb, C., Maguiña, C., \& González, E. (2010). Factores asociados a la presentación víscerohemolítica de loxoscelismo en el Hospital Nacional Cayetano Heredia, entre el 2000 y 2008. Acta Médica Peruana, 27(1), 29-36.

27. Zavaleta, A. (1987). Loxoscelismo, un problema de salud en el Perú. Boletín de la Oficina Sanitaria Panamericana, 103(4):378-86. 